\title{
Delayed Herniation of Coil Loop and Spontaneous Reposition in a Superior Cerebellar Artery Aneurysm
}

\author{
Ki Bum Sim, MD', Ji Kang Park, MD², O-Ki Kwon, MD³ , Jung Cheol Park, MD
}

Herniation of coil loops into the parent artery is one of the complications of endovascular embolization with detachable coils. In this clinical setting, we cannot predict the consequence of the herniated coil loop. We report an unusual case of a superior cerebellar artery (SCA) aneurysm with delayed coil herniation into the basilar artery and spontaneous reposition into the SCA.

Key Words : Cerebral aneurysm; Endovascular treatment; Coil herniation

Herniation of coil loops into the parent artery and associated thromboembolism is one of the serious complications during endovascular coil embolization of the cerebral aneurysm. Although herniation of one coil loop may not cause adverse events (1), we cannot exactly predict the consequence of herniated coil loop. We recently experienced a case of embolization of superior cerebellar artery (SCA) aneurysm in which delayed coil herniation developed into basilar artery (BA) and this coil loop was spontaneously repositioned into the left side SCA. Herein, we describe this case

Departments of ${ }^{1}$ Neurosurgery and ${ }^{2}$ Radiology, Jeju National University College of Medicine, Jeju, Korea; ${ }^{3}$ Department of Neurosurgery, Bundang Seoul National University Hospital, Sungnam, Korea; ${ }^{4}$ Department of Neurosurgery, Dongguk University Hospital, Goyang, Korea

Received July 25, 2010;

accepted after revision August 21, 2010.

Correspondence to: Ji Kang Park, MD, Department of Radiology, Jeju National University Hospital, Jeju National University College of Medicine, 1753-3, Ahra-1-dong, Jeju-si, Jeju Special Self-Governing Province 690-716, Korea.

Tel. 82-64-717-1371 Fax. 82-64-757-8276

E-mail: jkcontrast@naver.com

Neurointervention $2011 ; 6: 31-33$

This is an Open Access article distributed under the terms of the Creative Commons Attribution Non-Commercial License (http://creativecommons.org/licenses/by-nc/3.0) which permits unrestricted non-commercial use, distribution, and reproduction in any medium, provided the original work is properly cited. and discuss the clinical implication of this case.

\section{CASE REPORT}

A 34-year-old woman presented acute subarachnoid hemorrhage (Hunt and Hess grade 2) with severe headache. Left vertebral artery angiography demonstrated a $2.5 \mathrm{~mm}$ sized left side SCA aneurysm with a narrow neck (Fig. 1A). Under general anesthesia, we coiled this aneurysm by using a $2 \mathrm{~mm} \times 4 \mathrm{~cm}$ Microplex Hypersoft Coil V-trak system (MicroVention, California, USA) (Fig. 1B). The most proximal part of the detachment zone protruded minimally into the basilar artery (BA) after detachment (Fig. 1B, C). No thrombus formation was seen on angiogram. We performed follow-up fluoroscopy of coil mass to check out coil stability for 2 postoperative days (PODs), and there was no significant change of coil mesh (Fig. 1D). In the ninth POD, she complained newly developed headache and dizziness. Magnetic resonance imaging revealed a small size acute infarction in the left side SCA territory, but there is no evidence of recanalization of the aneurysm or recurred subarachnoid hemorrhage. Left side SCA was intact on MR angiography (MRA), however, fluoroscopy revealed unraveling of proximal coil loop and herniation into the BA (Fig. 1E). Herniated coil loop and remaining coil mesh were pulsating together with arterial pulsation. We initially 


\section{Ki Bum Sim, et al.}

considered stent placement to trap the coil loop, but decided to wait because she was stable and BA and its branches were intact on MRA. Oral aspirin $100 \mathrm{mg}$ per day was given. During following three days, we repeated fluoroscopy and ascertained no further herniation of the coil loop.

She was discharged and we repeated fluoroscopy weekly. Oral aspirin was maintained during follow-up period. In the 23rd POD, the shape of the herniated coil loop was changed and the herniated coil was spontaneously repositioned into the probable left side SCA on fluoroscopy (Fig. 1F). We considered that this configuration was more stable than previous one. We confirmed no change in configuration of the herniated coil loop on follow-up fluoroscopy during 3 months, and we performed MRA in the 90th POD. The previous infarction was resolved and there was no evidence of recanalization or SCA occlusion.

\section{DISCUSSION}

Coil loop herniation into the parent artery is an uncommon complication of aneurysm embolization, and the incidence was $2.4-4.2 \%$ in previous reports (1, $2)$. The mechanism of coil herniation appeared to be coil instability after detachment and excessive embolization (2). Contrarily, minimal protrusion of the coil's detachment zone into the parent artery may occur not infrequently during embolization, and this situation can be managed uneventfully with or without antiplatelet treatment. In our institution, we usually check follow-up fluoroscopy during two PODs in this situation. In our case, we could not indicate exactly the day when unraveling of coil developed, but probably it might occur near to ninth POD when the patient complained new headache and dizziness. Delayed unraveling and herniation of coil loop was reported

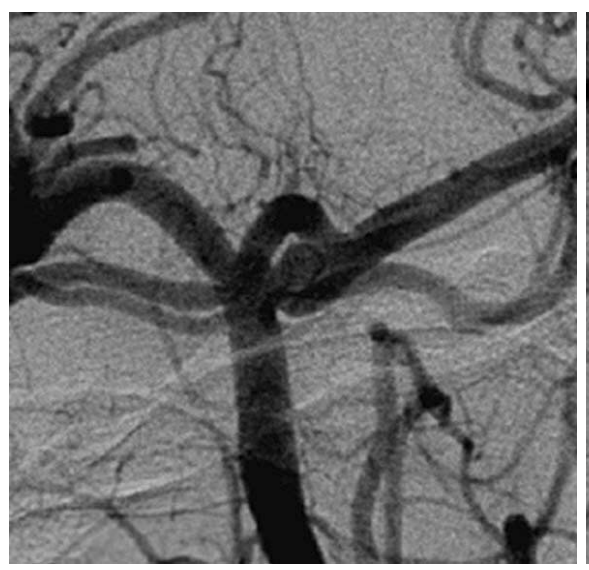

A

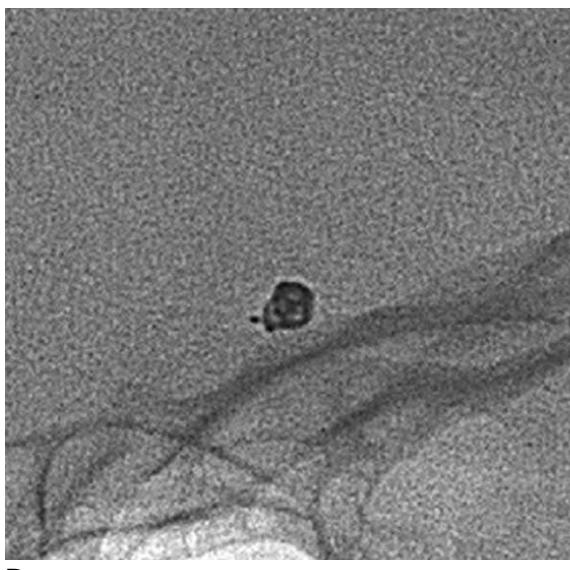

D

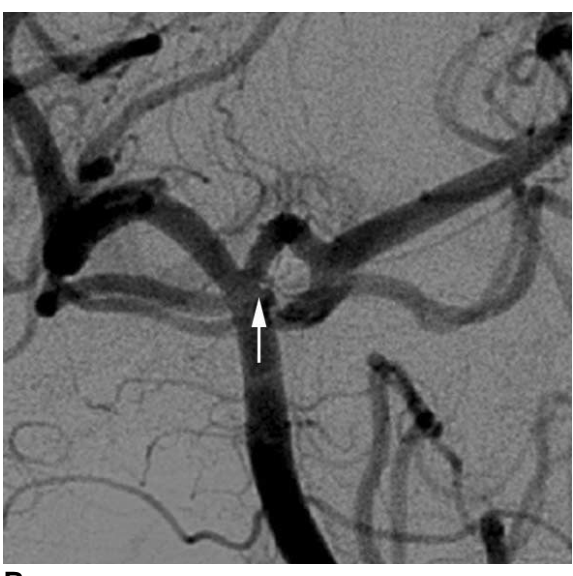

B

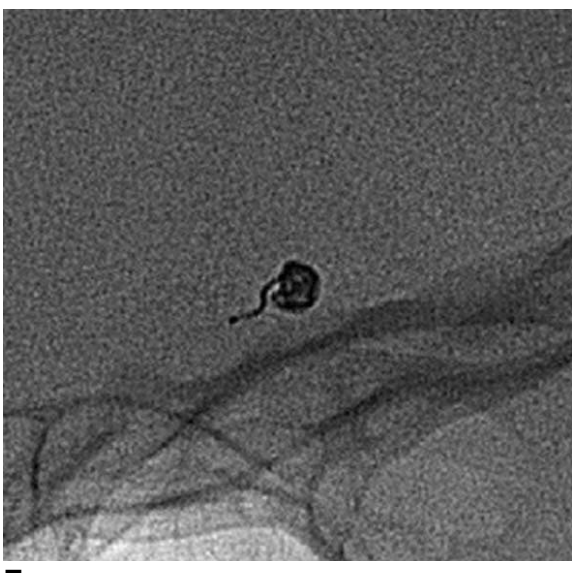

E

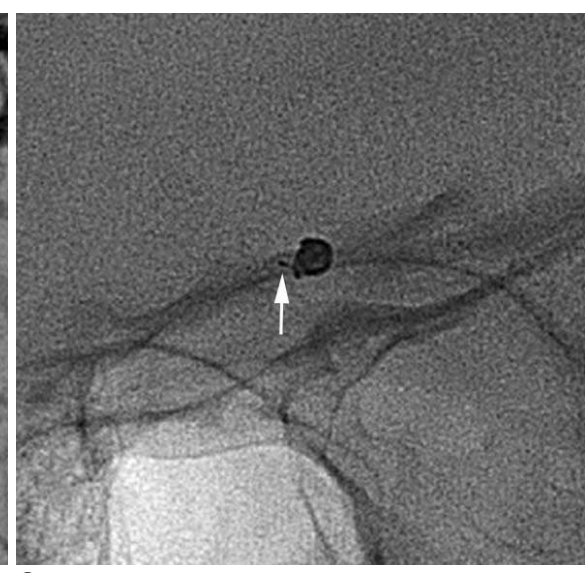

C

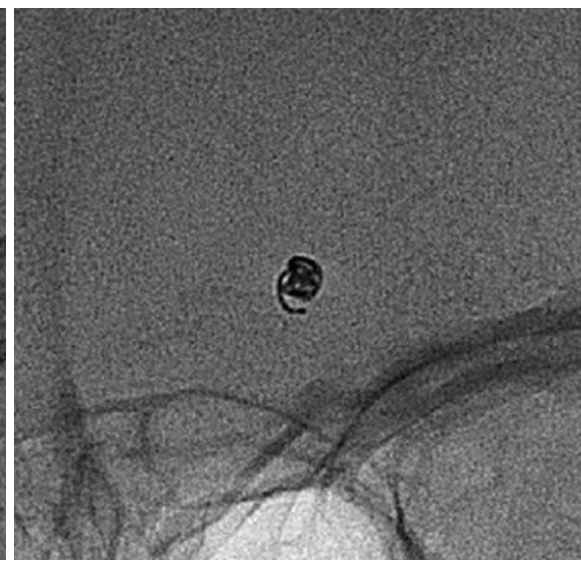

$\mathbf{F}$

Fig. 1. Working plane angiography reveals a $2.5 \mathrm{~mm}$ sized left side superior cerebellar artery (SCA) aneurysm (A). Final angiography and fluoroscopy show minimal protrusion of the detach-zone into the basilar artery (B, C, arrow). No significant interval change in the configuration of the coil mesh is seen on the fluoroscopy at the second postoperative day (POD) (D). Unraveling and herniation of coil is seen on the fluoroscopy at the ninth POD (E). Reposition of the herniated coil into the left side SCA is seen on the fluoroscopy at the 23rd POD (F). 
previously (3). In our case, it was difficult to insert last coil loop into aneurysm sac and we remained short detachment zone within the lumen of parent artery. This configuration is usually stable, but it may cause adverse consequence like our case. So-called 'stored tension' (3) in last coil loop might cause delayed unraveling. In our opinion, if minimal protrusion of the detach-zone into the parent artery is present, we should consider the possibility of delayed herniation of coil loop, and follow-up fluoroscopy for the evaluation of coil mesh may be necessary during minimum two postoperative weeks.

Managements of coil herniation after detachment are controversial and include removal by using a snare (4), repositioning by using balloon (5), or trapping of coil by using stent placement $(2,6-8)$. Occasionally, coil herniation is left in the lumen of parent artery with or without anti-platelet treatment if herniated coil aligns along the parent arterial wall or only a small closed loop is herniated (4). There was no systematic analysis about the fate and risky configuration of herniated coil loops in the parent arterial lumen. Only a suggestion was presented that herniated coil with a free end pulsating in the direction of blood flow may be a risky configuration (4). In our case, there might be two options for the management of the coil herniation. First, we might be able to remove the coil mesh by using a snare, but we should consider the state of the coil mesh with abundant thrombus and possible thromboembolism. Second option is the bailout stent deployment. We can stabilize the herniated coil loop along the wall of BA by the stent deployment; however, we should consider the procedure related complication and delayed in-stent stenosis (8). Because she was stable and relatively young, we deferred stent placement. In our opinion, the herniated coil loop may be affected by hemodynamics of the parent artery and coil's tendency toward its natural loop shape. In our case, this restoration tendency made the last stable configuration of the herniated loop. In our opinion, follow-up fluoroscopy with anti-platelet medication may be another option and guide proper decision for management of this situation.

In conclusion, minimal coil protrusion into the parent artery may undergo delayed further unraveling and herniation of a coil loop, and the shape of the herniated coil can be changed to favorable configuration. When we consider management of this condition, follow-up fluoroscopy may be helpful, and conservative management with anti-platelet medication may be one of the options of various treatments.

\section{References}

1. Renowden SA, Benes V, Bradley M, Molyneux AJ. Detachable coil embolisation of ruptured intracranial aneurysms: a single center study, a decade experience. Clin Neurol Neurosurg 2009;111:179-188

2. Luo CB, Chang FC, Teng MM, Guo WY, Chang CY. Stent management of coil herniation in embolization of internal carotid aneurysms. AJNR Am J Neuroradiol 2008;29:1951-1955

3. Phatouros CC, McConachie NS, Jaspan T. Post-procedure migration of Guglielmi detachable coils and Mechanical detachable spirals. Neuroradiology 1999;41:324-327

4. Dinc H, Kuzeyli K, Kosucu P, Sari A, Cekirge S. Retrieval of prolapsed coils during endovascular treatment of cerebral aneurysms. Neuroradiology 2006;48:269-272

5. Sugiu K, Martin JB, Jean B, Rufenacht DA. Rescue balloon procedure for an emergency situation during coil embolization for cerebral aneurysms. Technical note. J Neurosurg 2002;96:373376

6. Fessler RD, Ringer AJ, Qureshi AI, Guterman LR, Hopkins LN. Intracranial stent placement to trap an extruded coil during endovascular aneurysm treatment: technical note. Neurosurgery 2000;46:248-251

7. Schutz A, Solymosi L, Vince GH, Bendszus M. Proximal stent fixation of fractured coils: technical note. Neuroradiology 2005;47:874-878

8. Yoo E, Kim DJ, Kim DI, Lee JW, Suh SH. Bailout stent deployment during coil embolization of intracranial aneurysms. AJNR Am J Neuroradiol 2009;30:1028-1034 